\title{
Simulation and Modelling of Cathodic Protection Systems by the Finite Elements and the Boundary Elements Methods
}

\author{
C. Barrios Durstewitz, F. Almeraya-Calderón, R. Núñez Jaquez, C. Gaona \\ Tiburcio, A. Martínez Villafañe \\ Centro de Investigación en Materiales Avanzados, S.C. (CIMAV), División de Deterioro de \\ Materiales e Integridad Estructural / Grupo de Corrosión, Miguel de Cervantes No.120 \\ Complejo Industrial Chihuahua, C.P. 31109, Chihuahua, Chih., México
}

Received 19 April 2004; accepted 9 February 2004

\begin{abstract}
The development of mathematical models and the simulation of cathodic protection systems applied to buried tubing are presented. The solution of partial differential equations is obtained by numerical methods like finite elements and boundary elements. The purpose is developing a quantitative method of potential distribution prediction on the surface of the buried structure, covering aspects like selection of materials, geometric configuration and design of the system. As verification problems were used the problem of the square tank, and the problem of the cylindrical container. Finally two cases of buried structures are presented.
\end{abstract}

Keywords: cathodic protection, buried structures, modeling, simulation, numerical methods, fem, bem.

\section{Introducción}

En México una de las principales industrias es la del petróleo, la cual genera un alto ingreso económico a el país. Por esta razón es de suma importancia mantener en optimas condiciones su infraestructura, que entre otras cosas esta constituida por una intrincada red de tuberías y ductos que transportan diferentes productos,

\footnotetext{
* Corresponding author. E-mail address: carlos.barrios@cimav.edu.mx
} 
y están expuestos a ambientes agresivos como son bajo tierra y marinos que propician el fenómeno de la corrosión con el consecuente detrimento de sus propiedades útiles.

Los métodos de protección más empleados contra la corrosión en la práctica recurren a técnicas electroquímicas. Entre estos se encuentra la protección catódica, ya sea por ánodos de sacrificio o corriente impresa. En la actualidad en México se utilizan sistemas de protección catódica por corriente impresa en las tuberías bajo tierra que transportan hidrocarburos.

Una de las herramientas importantes para asegurar la buena integridad de los ductos y el desempeño de los sistemas contra la corrosión es la creación de modelos matemáticos confiables, que arrojen resultados lo más cercano posible a la realidad.

En el diseño convencional de sistemas de protección catódica se ignora la distribución de potenciales y densidad de corriente a través del electrolito y en la superficie de la estructura a proteger.

Durante los últimos treinta años se han desarrollado y aplicado técnicas de numéricas para simular diferentes aspectos del fenómeno de la corrosión; principalmente en lo que respecta a sistemas de protección catódica en ambientes marinos se han reportado una gran cantidad de trabajos [1, 2, 3, 4], y en menor grado en sistemas bajo tierra $[5,6,7]$. Todos estos trabajos se han llevado acabo principalmente en países como Inglaterra y Estados Unidos. En cuanto a México los trabajos reportados en la literatura han sido pocos y principalmente se limitan a la utilización del método de diferencias finitas para resolver las EDP del sistema. Es poco lo que se ha incursionado en México con la utilización de los métodos de elemento finito y elemento en la frontera en aplicaciones a simulación de sistemas de protección catódica; entre estas se encuentran la creación de un software llamado PROCATSIM [8] desarrollado en conjunto por la Facultad de Química y el Departamento de Metalurgia de la UNAM, que resuelve la ecuación de Laplace por el método de diferencias finitas. 


\section{Modelo matemático de un sistema de protección catódica}

La ecuación gobernante de la distribución de potenciales en electroestática es la ecuación de Laplace [1-5].

$$
\nabla^{2} P=0
$$

Teniendo como dominio $(\Omega)$ esta ecuación la totalidad del conductor o electrolito y una función $P=P(x, y, z)$ o $P=P(x, y)$ según se trate de $3-\mathrm{D}$ o $2-\mathrm{D}$, este se encuentra limitado por las condiciones de frontera $\Gamma_{i}$, donde $i=1 \ldots n$. Las condiciones de frontera más comunes que se pueden encontrar en el caso de la corrosión son las siguientes (ver Fig 1):

$$
\begin{gathered}
P=P_{o} \text { en } \Gamma_{1} \\
i=0 \text { en } \Gamma_{2} \\
P=f\left(i_{a}\right) \text { en } \Gamma_{3} \\
P=f\left(i_{c}\right) \text { en } \Gamma_{4}
\end{gathered}
$$

donde $i$ es la densidad de corriente $\left[\mathrm{A} / \mathrm{m}^{2}\right]$ y es calculada por medio de la ecuación

$$
i=\frac{1}{\rho} \Delta P
$$

$\rho$ es la resistividad del medio o electrolito y sus unidades son $\mathrm{Ohm}-\mathrm{cm}$. La corrosión es un proceso electroquímico, pero la ecuación de Laplace no contiene información acerca de las reacciones químicas entre los metales involucrados. La unión entre el potencial eléctrico y las reacciones químicas se establece mediante las condiciones de frontera 4 y 5 , reacciones anódica y catódica, respectivamente. 


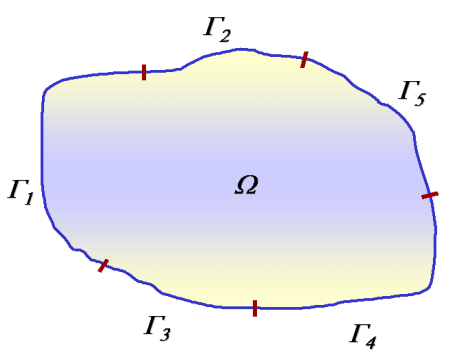

Figura 1. Dominio 2-D de solución y condiciones de frontera para la ecuación de Laplace.

La relación existente entre la densidad de corriente $\left(i_{a}, i_{c}\right)$ y el potencial $(P)$ se denomina en electroquímica como polarización. Esta función que describe la relación entre estas dos variables resulta en realidad por demás compleja, ya que depende de muchas propiedades tanto del electrolito como del metal, la interfase entre ambos y la temperatura. Por lo tanto la precisión que se puede obtener al simular procesos de corrosión depende principalmente de los datos utilizados en las condiciones 4 y 5 (curvas de polarización anódica y catódica). Existen en la literatura varios trabajos en los cuales se supone que la relación potencial contra densidad de corriente en estructuras bajo tierra esta compuesta por tres reacciones principalmente $[6,9,10]$. Estas son la disolución del Fe, la reacción de reducción del oxígeno y la reacción de reducción del hidrógeno. Donde la corriente total es la suma de las corrientes de cada una de las reacciones: $i=i_{F e}+$ $i_{O 2}+i_{H 2}$.

Para obtener la solución de las ecuaciones parciales que gobiernan el comportamiento de la distribución de potenciales y corriente, se recurre a métodos numéricos, debido principalmente a que una solución analítica de este tipo de problemas con condiciones de frontera lineales y geometrías complejas es por demás complicado o imposible. Los métodos numéricos generalmente empleados en estos casos son elemento finito y método de elementos de contorno. En el presente trabajo se utiliza el método de elemento finito a través del paquete ANSYS. La adaptación de las herramientas con las que cuenta dicho paquete computacional a los problemas de protección catódica. Para el caso del método de elementos de contorno, se recurre a un subrutina desarrollada en VB6, la cual utiliza elementos constantes para definir y simular el modelo, por el 
momento esta limitada a casos bidimensionales. Y los problemas tratados con esta, por consiguiente son en $2 \mathrm{D}$.

\section{Desarrollo}

La simulación con ANSYS / Multiphysics se llevó acabo con el siguiente ejemplo de un tanque cuadrado, comúnmente en la literatura [1-4]. Posteriormente se hizo otra simulación en ANSYS de una sección de tubería enterrada protegida por un ánodo de magnesio.

\section{Tanque cuadrado}

En este caso se conectan eléctricamente una placa de hierro y una barra de aluminio, que se encuentran sumergidos en agua de mar sintética $(\mathrm{NaCl} 3 \%)$. La geometría es un recipiente cuadrado considerando la profundidad como la unidad

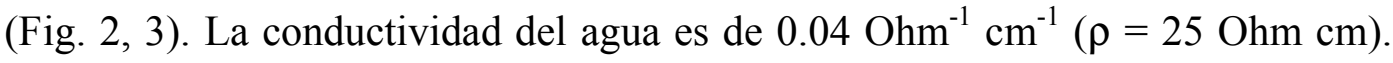
Se asume que la barra de aluminio tiene un potencial constante de $-686 \mathrm{mV}$ y la relación de polarización es no lineal.

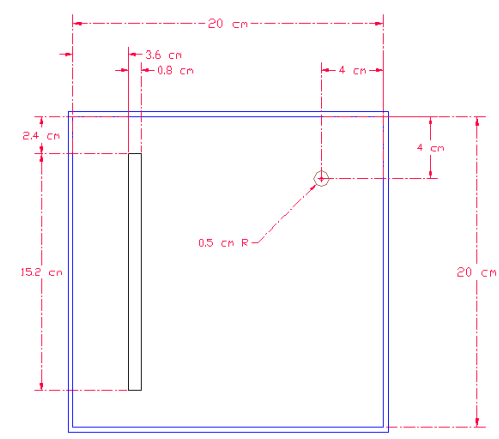

Figura 2. Geometría del problema del recipiente cuadrado.

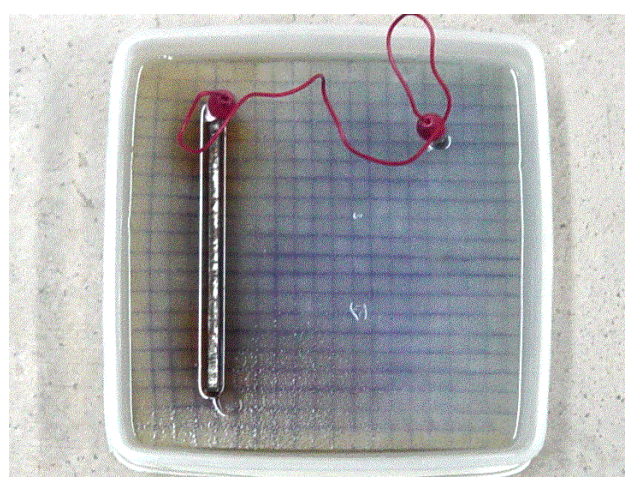

Figura 3. Arreglo experimental de par galvánico acero / aluminio.

\section{Recipiente cilíndrico}

Este problema consiste en determinar la distribución de potencial y densidad de corriente eléctrica. La configuración geométrica consiste de un recipiente de forma cilíndrica que contiene en el fondo dos metales diferentes (cátodo: Pt; ánodo: Fe), Fig. 4, los cuales están sumergidos en un electrolito (agua de mar 
sintética: solución de $\mathrm{NaCl}$ al 3\%) y en contacto eléctrico para formar un par galvánico. Se considera que la relación de polarización tiene un comportamiento lineal. Las dimensiones son $a=1 \mathrm{~cm}, c=2.5 \mathrm{~cm} \mathrm{y} b=3 \mathrm{~cm}$.

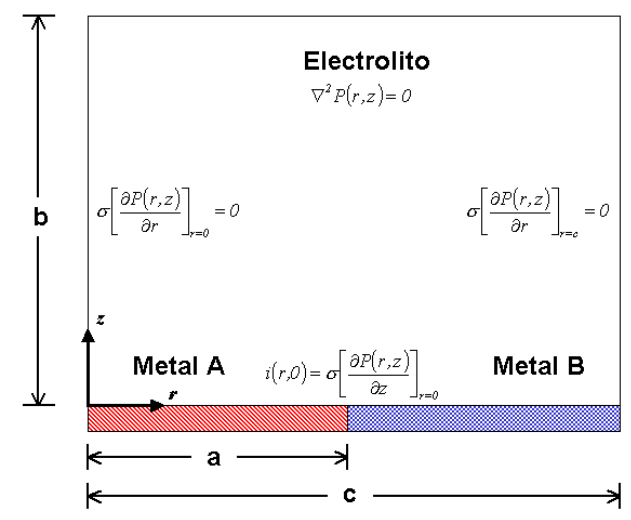

Figura 4. Geometría del recipiente cilíndrico.

\section{Tanque enterrado}

En este caso se tiene un tanque enterrado de acero, sin recubrimiento, con un diámetro de $3.048 \mathrm{~m}(10 \mathrm{ft})$, una longitud de $12.192 \mathrm{~m}(40 \mathrm{ft})$ a una profundidad de $0.6096(2 \mathrm{ft})$. Cuenta con un sistema de protección catódica por ánodos de sacrificio de $\mathrm{Mg}$, los cuales están a una distancia del tanque de $4.572 \mathrm{~m}$ (15 ft), el terreno circundante tiene una resistividad de $100 \mathrm{Ohm}-\mathrm{m}$. Se considera que la relación entre el potencial y corriente en la superficie del tanque tiene un comportamiento lineal, y los ánodos un potencial constante de $-1.7 \mathrm{~V}$. La geometría se muestra en la Fig. 5.

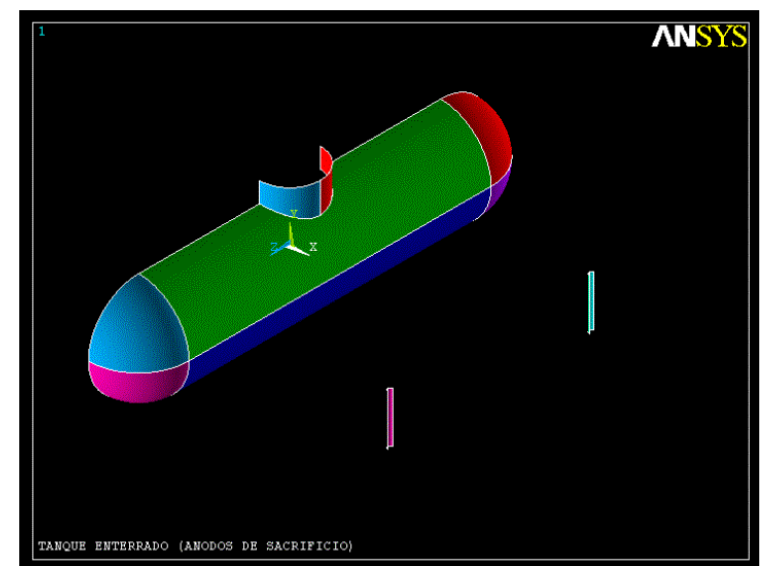

Figura 5. Geometría del tanque enterrado. 


\section{Tubería enterrada}

La geometría consisten en una tubería con un diámetro de 36 in $(0.9144 \mathrm{~m})$, una longitud de $50 \mathrm{~m}$ y a una profundidad de $2 \mathrm{~m}$, protegida por un sistema de protección catódica por corriente impresa y el recubrimiento presenta 3 defectos, que dejan al descubierto el metal del tubo. El medio presenta una resistividad de $100 \mathrm{Ohm}-\mathrm{m}$ y el lecho anódico se encuentra a una distancia de la tubería de 50 m. La cama de ánodos es tipo horizontal con una longitud de $15 \mathrm{~m}$, y a una profundidad de $2 \mathrm{~m}$. El objetivo del estudio es encontrar el potencial, con respecto a un electrodo de referencia de $\mathrm{Cu} / \mathrm{CuSO}_{4}$ saturado, en el lecho anódico para el cual el potencial en las zonas sin recubrimiento tenga el valor mínimo del criterio de protección $\left(-0.85 \mathrm{~V}\right.$ vs. $\mathrm{Cu} / \mathrm{CuSO}_{4}$ sat). La geometría de la tubería y el lecho anódico se presenta en la Fig. 6.

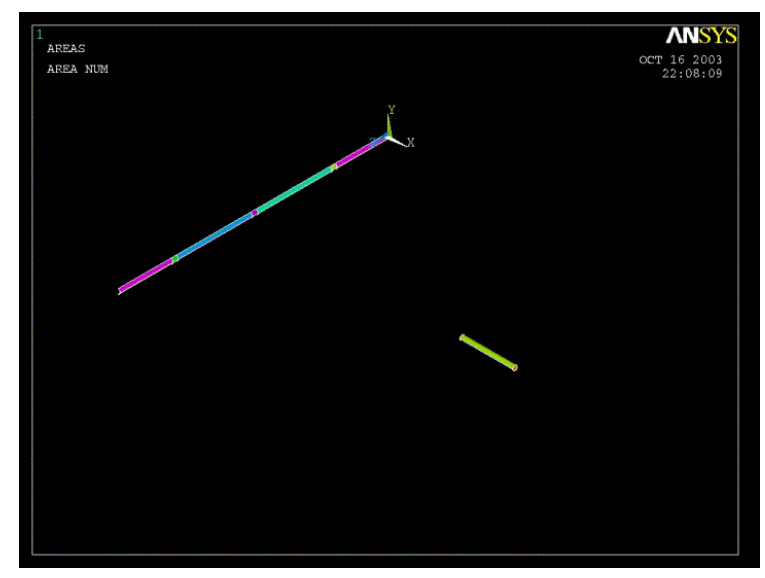

Figura 6. Geometría del la tubería enterrada. Los anillos que se ven sobre la tubería representan los defectos e el recubrimiento.

\section{Simulación}

La forma como se hace la simulación en ANSYS es utilizando su opción de análisis térmico, ya que existe una analogía entre las expresiones matemáticas que describen el comportamiento térmico de conducción de un sistema y el proceso de conducción eléctrica. La parte electroquímica se introduce en las condiciones de frontera, es decir las curvas de polarización, las cuales se pueden sustituir como condiciones de contorno de un proceso de transferencia de calor convectivo. 
Si consideramos en la ecuación de Laplace la variable de campo $P$ como la temperatura $T$, y la variable $i$ como el flux de calor $q$, así como la conductividad del medio $(1 / \rho)$ por la conductividad térmica $(k)$, obtenemos la ecuación que describe el comportamiento de la temperatura en el fenómeno de transferencia del calor. La transferencia de calor se lleva acabo por 3 mecanismos: conducción, convección y radiación. Centrando la atención en el mecanismo de convección, el flux de calor $(q)$ lo determina la siguiente expresión:

$$
q=h(\Delta T)
$$

dónde $h$ es el coeficiente de película. Para realizar la analogía al fenómeno de corrosión se hace a través de una expresión que relacione la densidad de corriente y el potencial, que haciendo una comparación con la expresión con el mecanismo de convección resulten análogas. En base a lo anterior se puede simular mediante ANSYS/ MULTIPHYSICS problemas de corrosión, en el caso de la condición de frontera por convección se puede utilizar la siguiente expresión:

$$
i=\frac{1}{\rho W}\left(P-P^{o}\right)
$$

el equivalente al coeficiente de película es $h=1 / \rho W$, un pseudo-coeficiente de película, donde $\rho$ es la resistividad del medio y $\mathrm{W}$ es el número de Wagner. Dicho número es la razón de la resistencia cinética entre la resistencia ohmica. Es la razón de la pendiente de polarización (evaluada en el sobre potencial de interés) dividido por la longitud característica y la resistencia de la solución:

$$
W=\left(\frac{\kappa}{L}\right)\left(\frac{\partial E}{\partial i}\right)=\frac{R_{p}}{R_{\Omega}}
$$

donde $\kappa($ ó $1 / \rho)$ es la conductividad de la solución (ohm-m) $)^{-1}$ y L es una longitud característica (m), o la dimensión de irregularidad. Se puede tomar la derivada parcial, $\partial \mathrm{E} / \partial \mathrm{i}$, de cualquier expresión analítica que describa la función entre $\mathrm{E}$ e 
$\mathrm{I}$, $\mathrm{u}$ obtener una pendiente de polarización, $\mathrm{R}_{\mathrm{p}}$, de datos experimentales de $\mathrm{E}$ vs. $\mathrm{I}$. Tal pendiente puede ser evaluada en la densidad de corriente promedio o en cualquier densidad aplicada. La derivada parcial tiene unidades de Ohm- $\mathrm{m}^{2}$. Los números de Wagner resultantes para varias expresiones analíticas se muestran en la tabla 1.

La simulación en base al método de elementos de contorno, se hizo con una subrutina creada en VB6 y se limito a los problemas de el tanque cuadrado y el recipiente cilíndrico, ya que únicamente soporta elementos unidimensionales, esto es para casos coplanares.

\section{Tabla 1}

\begin{tabular}{lccc}
\hline \hline $\begin{array}{l}\text { Distribución } \\
\text { de corriente }\end{array}$ & $\begin{array}{c}\text { Relación entre sobrepotencial y } \\
\text { densidad de corriente } E=f(i)\end{array}$ & $\begin{array}{c}\text { Término de } \\
\text { resistencia a la } \\
\text { polarización }(\partial \mathrm{E} / \partial \mathrm{d})\end{array}$ & $\begin{array}{c}\text { Numero de } \\
\text { Wagner }\end{array}$ \\
\hline Primaria & $\ldots$ & $\ldots$ & $\frac{\kappa}{L}$ \\
Secundaria & $E=B i($ Lineal $)$ & $\frac{\kappa B}{L}$ \\
Secundaria & $E=B^{\prime} \ln \left(\frac{i}{i_{\text {corr }}}\right)($ Tafel $)$ & $\frac{B^{\prime}}{i}$ & $\frac{\kappa B^{\prime}}{i L}$ \\
Terciaria & $E=\left(\frac{R T}{n F}\right) \ln \left(1-\frac{i}{i_{L}}\right)($ Difusión $)$ & $\left(\frac{R T}{n F}\right) \cdot\left(\frac{1}{i_{L}-i}\right)$ & $\frac{\kappa R T}{\left[n F\left(i_{L}-i\right) L\right]}$ \\
\hline \hline
\end{tabular}

\section{Resultados}

Los resultados obtenidos para cada uno de los casos se muestran a continuación.

\section{Recipiente cilíndrico}

En este caso, tanto la simulación con ANSYS como con la rutina muestra un buen grado de concordancia (Fig. 7 y 8). En la Fig. 8 la grafica muestra el comparativo de los potenciales naturales en la superficie de cada uno de los metales inmersos en la solución sin existir una conexión electrónica entre 
ambos. Al establecerse el contacto electrónico se forma un par galvánico, y los potenciales en la superficie se modifican como consecuencia del la polarización; el potencial anódico sufre una variación a valores más positivos, mientras que el metal cátodo, se mueve hacia valores más negativos y en la interfase entre ambos se presenta una atenuación de los potenciales (transición). La distribución de corriente, en base a la distribución de potenciales, es de tipo secundario, ya que las líneas de isopotencial en la superficie de los metales presentan una cierta inclinación. El gradiente de potencial tiende a ser mayor en la interfase de los dos metales, y es aquí donde se presenta la mayor densidad de corriente.
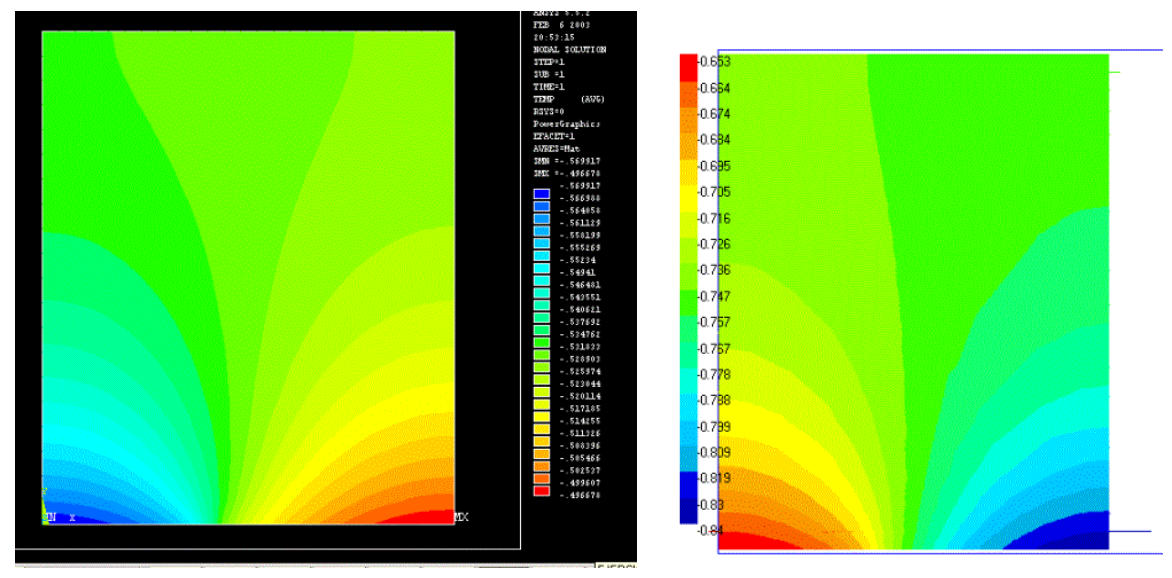

Figura 7. Distribuciones de potencial en el recipiente cilíndrico. La figura del lado izquierdo se obtuvo con el paquete ANSYS y el de la derecha con la subrutina por elementos de contorno.

\section{Recipiente cuadrado}

Las soluciones obtenidas mediante los métodos de elemento finito y elementos de contorno para el caso del tanque cuadrado, tuvieron un buen grado de ajuste con el problema experimental, como se puede observar en la Fig. 9, donde se observa la comparación entre la distribución de potenciales calculados y experimentales. En la Fig. 10 se muestra la distribución de potenciales obtenida por el método de elemento de contorno y el perfil de potenciales de la cara de la 

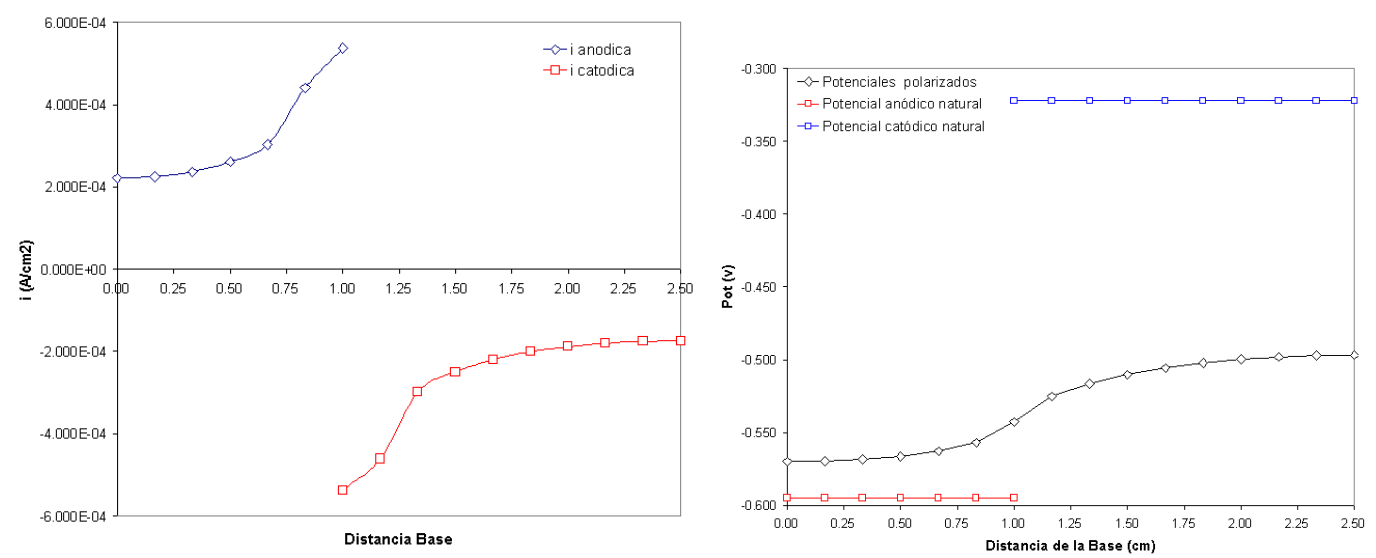

Figura 8. La figura izquierda muestra la densidad de corriente en la superficie de cátodo y ánodo. La del lado derecho las comparaciones entre los potenciales naturales y polarizados de la superficie de los metales.
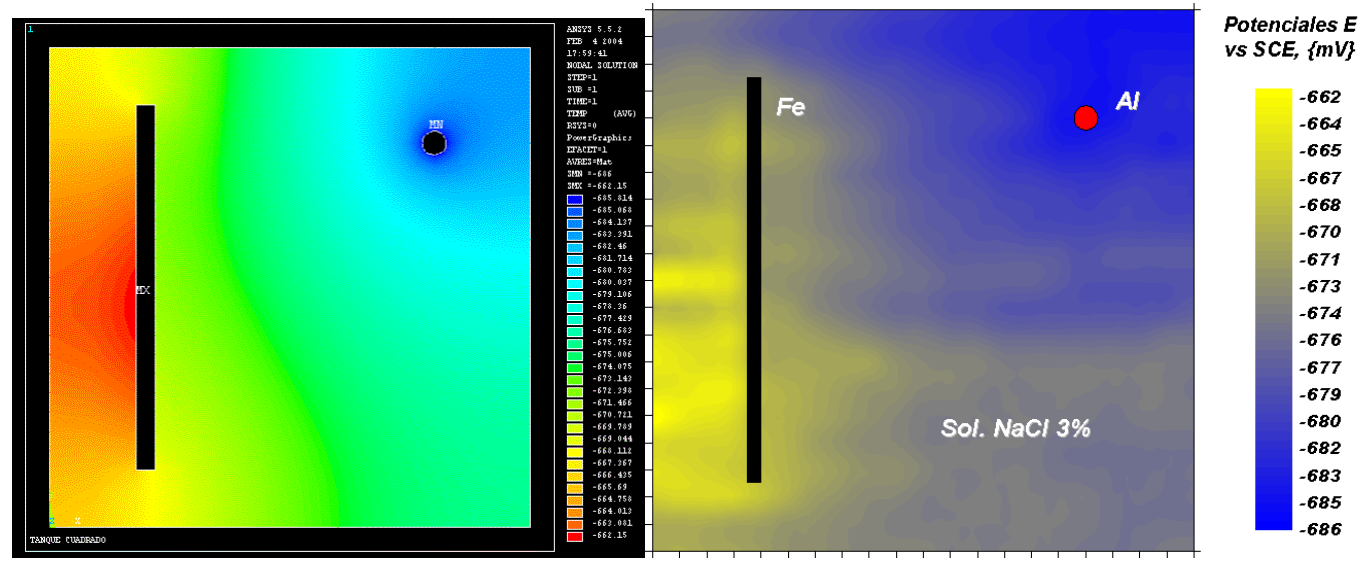

Figura 9. En la figura del lado izquierdo se muestra la distribución de potenciales calculados por ANSYS; en la figura derecha se ve la distribución de potenciales experimentales.

placa que esta frente al ánodo de aluminio. Sobre la superficie del aluminio se tiene una distribución de corriente primaría, esto es la línea de isopotencial es paralela a la superficie del aluminio, como era de esperarse, ya que fijo como condición de frontera un potencial constante en el ánodo. Se observa también en la placa de Fe que la zona opuesta a la barra de aluminio presenta los valores más positivos, indicativo de que en esa zona la densidad de corriente es menor. En base a los gradientes de potencial se puede decir que los valores de densidad más altos se encuentran en las esquinas de la placa, al estar las líneas de isopotencial 
más cercanas entre sí. La distribución de corriente sobre la placa de Fe presenta una distribución de corriente de tipo secundario; esto se puede ver en las líneas de isopotencial, presentan una cierta inclinación respecto a la superficie de la placa.
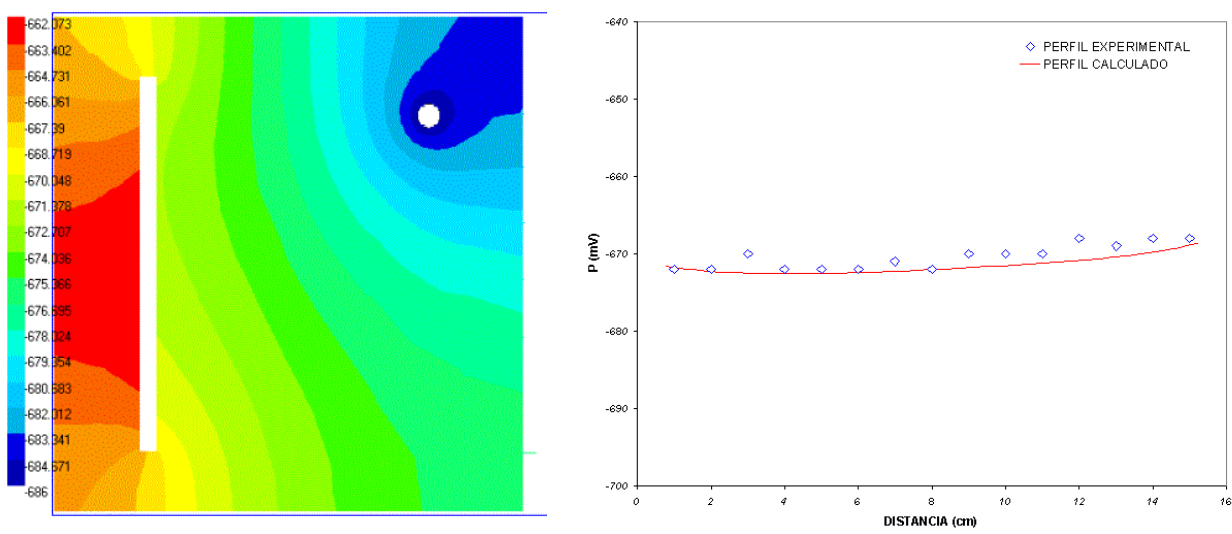

Figura 10. En la figura de la izquierda se muestra la distribución de potenciales obtenida por la subrutina del método de elementos de contorno. En la de la derecha el perfil de potenciales en la cara de la placa de acero frente al ánodo de Al obtenida por el método anterior.

\section{Tanque enterrado}

Para este caso únicamente se hicieron los cálculos por medio del paquete ANSYS. La parte que se modelos consistió en la porción de tierra que se encuentra entre los ánodos de Mg y en la superficie del tanque, Fig. 11. Se puede observar que la distribución de potenciales sobre la superficie es característica de una distribución de corriente secundaria, y los valores mas positivos de potencial se encuentran ubicados en la región cercana a la "boca" del tanque, específicamente en los vértices de la parte superior. Al ser los extremos del tanque redondeados favorece una distribución de la corriente más uniforme, ya que al existir esquinas rectangulares, la corriente tiende a concentrarse en esas partes, como se puede ver que sucede en el recipiente cuadrado. 

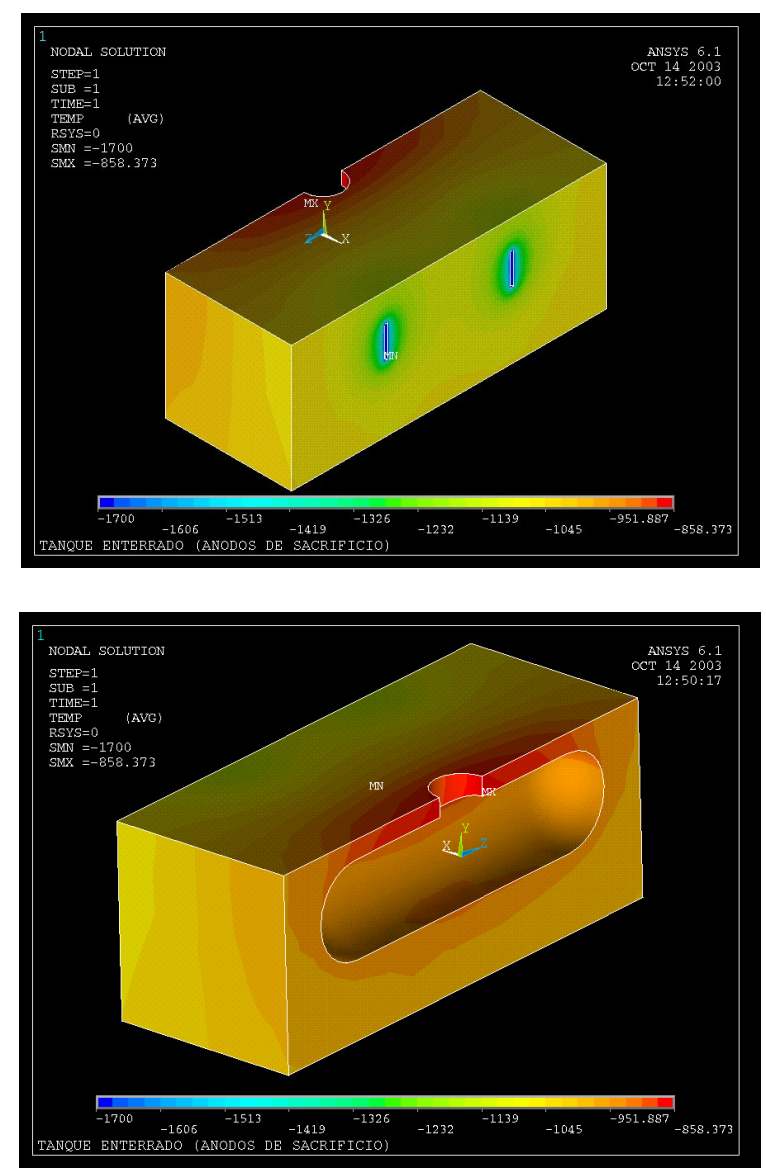

Figura 11. Distribución de potenciales para el tanque enterrado, obtenidos mediante ANSYS.

\section{Tubería enterrada}

Nuevamente el modelo por elementos finitos fue la porción de terreno entre la tubería y el lecho anódico, abarco una altura de $5 \mathrm{~m}$ desde el nivel del suelo hasta 3 metros por debajo de la tubería; en este caso se despreció el efecto dela resistencia de la tubería, ya que la simulación únicamente se llevó acabo en el dominio del terreno, para conocer la distribución de los potenciales y el potencial del lecho que cumpliera con la condición de un valor de $-850 \mathrm{mV}$ vs. $\mathrm{Cu} / \mathrm{CuSO}_{4}$ en la superficie de los defectos del recubrimiento. El valor necesario para alcanzar esto en el lecho anódico es de $-1 \mathrm{~V}$. La distribución de corriente en la superficie del la tubería (en los defectos) es de tipo secundaría, Fig. 12. 

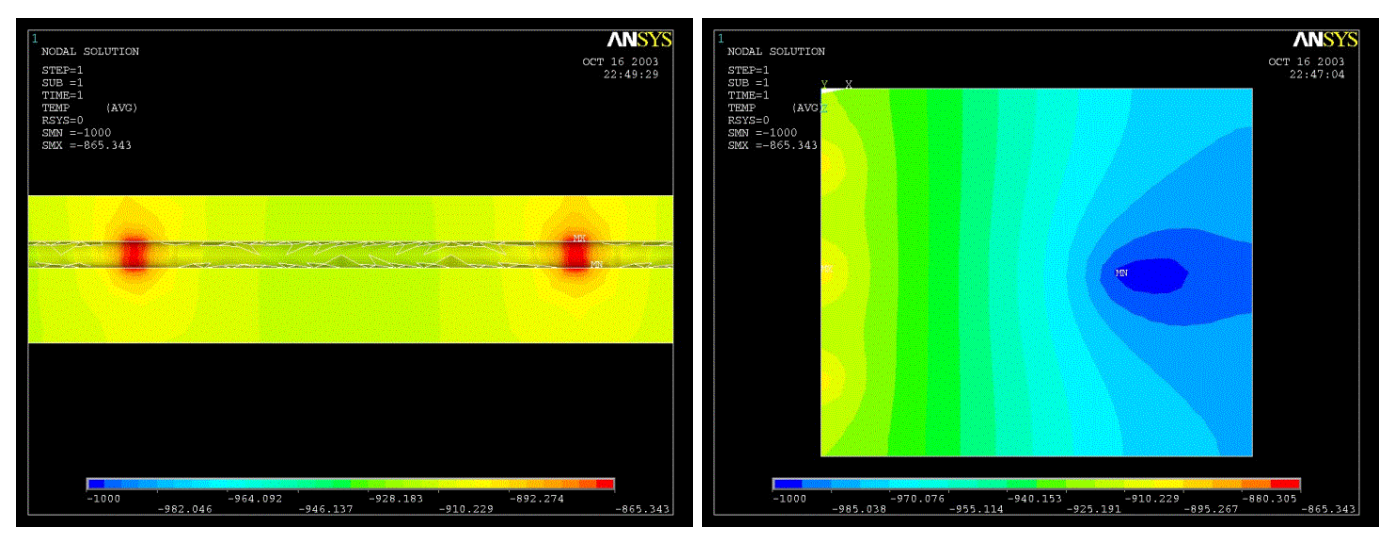

Figura 12. Distribución de potenciales para el caso de la tubería enterrada. La figura derecha se aprecia el detalle sobre los defectos, al tener el lecho anódico un potencial de $-1 \mathrm{~V}$. En la figura izquierda se muestra la distribución de potenciales sobre el terreno.

\section{Conclusión}

Se determinaron las ecuaciones parciales gobernantes para un sistema de protección catódica así como sus condiciones de frontera.

La solución de las ecuaciones parciales gobernantes del sistema se hacen a través del método numérico de elemento finito. Haciendo una analogía entre transferencia de calor y conducción de eléctrica en un electrolito se encontró una forma de simular procesos de corrosión mediante la utilización de la opción de análisis térmico del paquete ANSYS /Multyphysics.

Se creo una subrutina en basea VB6 para calcular la ecuación de Laplace bajo las condiciones de frontera de $\mathrm{E}=\mathrm{f}(\mathrm{i})$, teniendo como parámetros el numero de Wagner o Rp. mediante el método de elementos en la frontera con elementos constantes, para casos 2D. 


\section{Simulación y Modelación de Sistemas de Protección Catódica por los Métodos Numéricos de Elementos Finitos y Elementos de Contorno}

\section{Resumen}

En este trabajo se presenta el desarrollo de modelos matemáticos y simulación de sistemas de protección catódica aplicados a tuberías enterradas. La solución de las ecuaciones diferenciales parciales se obtiene mediante los métodos numéricos de elementos finitos y elementos de contorno. Todo esto con la finalidad de desarrollar un método cuantitativo de predicción de la distribución del potencial y corrientes en el medio y la superficie de la estructura a proteger, cubriendo los siguientes aspectos: disminución de los efectos de corrosión debidos a la selección de materiales, configuración geométrica y diseño del sistema. Como problemas de verificación se utilizaron los dos ejemplos mas conocidos: el problema del tanque cuadrado, y el problema del recipiente cilíndrico con el par ánodo / cátodo circulares y concéntricos.

\section{Referencias}

1. R.A. Adey and S.M. Niku, ASTM STP 1154, R.S. Munn, Ed. ASTM, 1992, pp. 248-264.

2. V.G. De Giorgi, E.D. Thomas, and A.I. Kaznoff, ASTM STP 1154, R.S. Munn, Ed., ASTM, Philadelphia, USA, 1992, pp .265-276.

3. R.D. Strommen, ASTM STP 1154, R.S. Munn, Ed. ASTM, 1992, pp. 229247.

4. R.S. Munn, Computer Modeling in Corrosion, ASTM STP 1154, R.S. Munn, Ed. ASTM, 1992, pp. 215-228.

5. K.J. Kennelley, L. Bone, M.E. Orazem, Corrosion 49(3) (1993) 199-210.

6. M.E. Orazem, J.M. Esteban, K.J. Kennelley, R.M. Degerstedt, Corrosion 53(4) (1997) 264-272.

7. M.E. Orazem, J.M. Esteban, K.J. Kennelley, R.M. Degerstedt, Corrosion 53(6) (1997) 427-436.

8. J. Amador, J. Genesca, Afinidad 59(501) (2002) 530-541.

9. W. Sun, K.M. Liu, J. Electrochemical Soc. 147(10) (2000) 3687-3690.

10. L.S. Xiao-Zhe, J. Newmann, J. Electrochemical Soc. 148 (4) B (2001) 157-B162. 